\title{
BIOSORCIÓN SIMULTANEA DE PLOMO Y CADMIO EN SOLUCIÓN ACUOSA POR BIOMASA DE HONGOS Penicillium sp.
}

\section{SIMULTANEOUS BIOSORPTION OF LEAD AND CADMIUM FROM AQUEOS SOLUTION BY FUNGAL BIOMASS Penicillium sp.}

\author{
Juan G. Sánchez*, José L. Marrugo', Iván D. Urango \\ Recibido para publicación: Noviembre 27 de 2013 - Aceptado para publicación: Mayo 23 de 2014
}

\section{RESUMEN}

La descarga de metales pesados en el ambiente genera impactos significativos en la salud humana y ciclos biológicos. Algunos microorganismos son conocidos por su alta capacidad de adsorción de metales, entre estos los hongos. El objetivo del presente trabajo fue evaluar la capacidad de remoción de $\mathrm{Pb}$ (II) y Cd (II) en soluciones acuosas con hongos Penicillium sp., aislando la cepa fúngica del suelo de la mina el Alacrán (CórdobaColombia) y determinando la influencia en las variables $\mathrm{pH}$, concentración inicial de metales y temperatura, analizando las isotermas de Freundlich y Langmüir, Verificando los grupos funcionales que intervienen en la adsorción por análisis infrarrojo (IR), con el fin potencializar su uso en el saneamiento de aguas residuales. Los análisis se realizaron empleando los equipos Thermo scientific de absorción atómica modelo ICE 3000 serie y espectrofotómetro FT-IR Nicolet Is5 realizando control de calidad con material de referencia de Pb y Cd SRM1643e. A 51,5 $\mathrm{mg} \mathrm{L}^{-1}$ de $\mathrm{Pb}$ y Cd se pudo encontrar los mayores porcentajes de remoción para 0,5 g de biomasa. Las máximas adsorciones se dieron a $\mathrm{pH}$ ácido (4-5) y $60{ }^{\circ} \mathrm{C}$, logrando remociones del $92,4 \%$ para $\mathrm{Pb}$ y $80 \%$ para Cd. Las isotermas se ajustan mejor al modelo Langmuir. El análisis IR muestra grupos - $\mathrm{OH},-\mathrm{NH}, \mathrm{C}-\mathrm{N}, \mathrm{C}-\mathrm{H}$, $\mathrm{N}-\mathrm{H}, \mathrm{C}=\mathrm{O}$, amida I y amidas II y polisacáridos, atribuyendo esto la atracción en los metales y la biomasa fúngica.

Palabras Clave: Adsorción de metales, Metales pesados, hongos, Penicillium sp.

\begin{abstract}
The discharge of metals in the environment generates significant impacts on human health and the life cycles of living organisms. Some microorganisms are known for their high adsorption capacity for metals, including fungi. The aim of this study was to evaluate the remotion capacity of $\mathrm{Pb}$ (II) and $\mathrm{Cd}$ (II) in aqueous solutions with Penicillium sp. fungi, isolating the soil fungal strain of the Alacrán Mine (Cordoba-Colombia) and determining the influence of the removal on the variables $\mathrm{pH}$, metal concentration and temperature, analyzing the Langmuir and Freundlich isotherms, verifying the functional groups involved in the adsorption by infrared analysis (IR)., to potential their use in wastewater treatment. Analyses were conducted using Thermo Scientific equipment,
\end{abstract}

'Universidad de Córdoba, Grupo de Investigación en aguas, Química Aplicada y Ambiental - GAQAA, Montería-Colombia, Departamento de Química. Carrera 6 No. 76 -103 Montería - Colombia, Tel (261) 7860577 Fax: (261) 7860577 Email: JGSC09@hotmail.com. 
atomic absorption ICE model 3000 Series and FT- IR Nicolet IS5 spectrophotometer. Quality control was performed for metal analysis with reference material SRM 1643e. The results indicate that to the concentration of $51.5 \mathrm{mg} \mathrm{L}^{-1}$ showed higher removal percentages for $0.5 \mathrm{~g}$ of biomass. The maximum adsorption occurred at acidic $\mathrm{pH}(4-5), 60{ }^{\circ} \mathrm{C}$ and $2 \mathrm{~g}$ of biomass, achieving removals of $92.4 \%$ for $\mathrm{Pb}$ and $80 \%$ for $\mathrm{Cd}$. Isotherm analyzes best fit the model Langmuir. IR analysis shows $-\mathrm{OH},-\mathrm{NH}, \mathrm{C}-\mathrm{N}, \mathrm{C}-\mathrm{H}, \mathrm{N}-\mathrm{H}, \mathrm{C}=\mathrm{O}$, amide I and amide $\mathrm{II}$ and polysaccharides, attributing this attraction in metals and fungal biomass.

Key words: metal adsorption, heavy metals, fungi, Penicillium sp.

\section{INTRODUCCIÓN}

La creciente actividad industrial es culpable en gran parte de los principales problemas de contaminación ambiental y del daño en los ecosistemas, generando la acumulación de contaminantes tóxicos como los metales pesados cromo, cobre, plomo, cadmio, cinc, níquel, mercurio, etc. (Pino et al. 2006) y causando daños en la salud humana, por ejemplo, el plomo $(\mathrm{Pb})$ es uno de los metales pesados más tóxicos, causando anemia, encefalopatía, hepatitis y síndrome nefrítico (Deng et al. 2006). La exposición a cadmio (Cd) genera riesgos severos a la salud humana, puede provocar cáncer, daño renal, destrucción de la membrana mucosa, vómito, diarrea, daño óseo, y la enfermedad de itai-itai, así como también afecta la producción de progesterona y testosterona (Johannes et al. 2006).

Existen varias tecnologías para remediar la carga de metales pesados de agua residual industrial, en donde solo la adsorción es viable a escala industrial en cuanto a la relación costo-beneficio (Lin et al. 2008). Sin embargo, el precio elevado de adsorbentes (usualmente carbón activado) es considerado como el principal obstáculo para la aplicación industrial. Por eso el foco de estudio de adsorción de metales pesados ha estado inclinado hacia materiales naturales que están disponibles en cantidades extensas (Agarwai et al. 2006). Se han publicado estudios en relación con el posible uso del hongo Penicillium sp., como un potencial bioadsorbente de metales pesados por su extraordinaria capacidad de adsorción extracelular (Fan et al. 2008).

Por lo antes indicado, el objetivo de este trabajo fue evaluar la capacidad de remoción simultanea de los metales $\mathrm{Pb}$ (II) y $\mathrm{Cd}$ (II) en soluciones acuosas por medio de hongos Penicillium sp., aislando la cepa fúngica del suelo de una mina de alta carga de contaminantes como la mina el Alacrán (Córdoba-Colombia), determinando la influencia en la remoción de las variables $\mathrm{pH}$, concentración inicial de metales y temperatura de medio. Verificando los grupos funcionales activos de la biomasa fúngica que intervienen en el fenómeno de adsorción por medio de análisis infrarrojo (IR), analizando los modelos de adsorción de isotermas Langmuir y Freundlich.

\section{MATERIALES Y MÉTODOS}

\section{Muestreo y caracterización de cepas fúngicas de Penicillium sp}

Las muestras de suelo fueron colectadas en enero del 2013, en la mina el Alacrán (CórdobaColombia), localizada en las coordenadas 
planas Norte $=1 ' 347.808$ y Este $=817.222$. Se tomaron diez muestras al azar a 330 metros de la entrada de la mina, excavando con una pala estéril aproximadamente 0,5 m tomando aproximadamente $500 \mathrm{~g}$ de suelo, se homogenizaron las muestras de cada punto en un recipiente plástico estéril, separando cerca de $500 \mathrm{~g}$ en fundas estériles herméticas para transportar al laboratorio (Valencia 2004).

Para realizar el aislamiento de la cepa fúngica, se tomó $1 \mathrm{~g}$ de muestra de suelo, que fue disuelta en agua bidestilada esterilizada. Se utilizaron placas ( $\mathrm{pH}$ 5) de Agar Czapek-Dox (CDA) con suplementos de sulfato estreptomicina (25 $\mathrm{mg} / 100 \mathrm{~mL}$ ) y ciclohexamida $(6 \mathrm{mg} / 100 \mathrm{~mL})$ para el aislamiento de hongos tolerantes de metales (Natarajan et al. 2010). De la muestra diluida de suelo, se tomó $0,1 \mathrm{~mL}$ inoculando en placas Agar CDA. Las placas inoculadas se incuban a $25^{\circ} \mathrm{C}$ durante siete días, tomando placas control con inoculaciones de agua bidestilada esterilizada, incubadas en las mismas condiciones.

Después del período de incubación, la especie de hongo crece en las placas y se transfieren a placas Agar CDA encubándose hasta que el crecimiento se haya completado. A partir de los cultivos de crecimiento en medios Agar CDA de siete días de crecimiento, las muestras fúngicas cultivadas se dividen y se observan características macroscópicas y microscópicas, de acuerdo a Valencia (2004). El cultivo fúngico puro se mantiene tanto en placas de Agar de CzapekDox como en caldos. El crecimiento masivo del hongo se realizó en medio líquido Czapek-Dox por treinta días. La biomasa generada de hongos Penicillium sp. se extrae por tamizado, siendo el material base de los experimento de biosorción (Natarajan et al. 2010).

\section{Diseño experimental}

Se utilizó un diseño experimental por bloques de un solo factor, donde la respuesta a medir fue la concentración de los iones metálicos en el equilibrio. Para cada factor en particular (Concentración inicial de metales, pH y temperatura) se mantuvieron, las demás variables fueron constantes, estrategia fuertemente recomendable si se supone que la variabilidad de los factores es alta. Los resultados de los análisis son presentados por la media $(\bar{X}) \pm$ desviación estándar (DS) de las determinaciones por duplicado. Para todos los análisis estadísticos el criterio de significancia se estableció a $\mathrm{p}<0.05$. El tratamiento de los resultados se realizó mediante el paquete estadístico Excel 2007 (Microsoft Office).

\section{Experimentos de Biosorción}

Se preparó una solución madre mixta de $\mathrm{Pb}$ (II), y Cd (II) a $1000 \mathrm{mg} \mathrm{L}^{-1}$, a partir de reactivos marca Merck. Todos los experimentos de biosorción se llevan a cabo con la misma solución madre (Natarajan et al. 2010). El efecto de la concentración de metales se determinó usando $100 \mathrm{~mL}$ de un stock múltiple de $\mathrm{Pb}$ (II) y Cd (II) a 8,0; 30,0; 51,5 y 103,0 $\mathrm{mg} \mathrm{L}^{-1}$ (preparado a partir de la solución madre) y 0,5 $\mathrm{g}$ de biosorbente a $\mathrm{pH} 4$ y $30^{\circ} \mathrm{C}$. Se incubaron en frascos agitando a $200 \mathrm{r} / \mathrm{min}$ durante 24 horas, después del período de incubación, los sobrenadantes se recogen por centrifugación a $6000 \mathrm{r} / \mathrm{min}$ durante 30 min. Luego la concentración de metales es determinada en el sobrenadante por absorción atómica con atomización electrotermia. El efecto de la concentración inicial arrojó los datos necesarios para el análisis de isotermas Freundlich y Langmüir. El efecto de $\mathrm{pH}$ se evalúa preparando un stock mixto de $\mathrm{Pb}$ (II) y Cd (II) a 51,5 mg/L y 0,5 g de biosorbente y el 
$\mathrm{pH}$ de las soluciones fue de 2, 3, 4, 5, 6, 7, 8, 9 y 10.

El efecto de la temperatura se determinó usando stock múltiple de $\mathrm{Pb}$ (II) y $\mathrm{Cd}$ (II) a 51,5 $\mathrm{mg} \mathrm{L}^{-1}$ y 0,5 g de biosorbente a $\mathrm{pH}$ 4. Las muestras se incubaron en frascos agitando a $200 \mathrm{r} / \mathrm{min}$ durante 24 horas a 20, 25, 30, 35, 40, 45, 50, 55 y $60^{\circ} \mathrm{C}$, respectivamente. Cada uno de los ensayos se realizó por duplicado, Después del período de incubación, el sobrenadante se recogió y analizó como lo describe en el efecto concentración inicial (Natarajan et al. 2010). Los análisis de metales se realizaron usando un espectrómetro marca THERMO SCIENTIFIC modelo ICE 3000 Serie, con una longitud de onda de 217 y 228,8 nm para $\mathrm{Pb}$ y $\mathrm{Cd}$, respectivamente.

\section{Análisis experimental de datos}

La cantidad adsorbida de Pb (II) y Cd (II) por la biomasa fue calculada usando la ecuación 1.

$$
q_{e}=\frac{\left(C_{0}-C_{e}\right)}{W} V
$$

Ecuación 1.

Donde $\mathbf{q}_{\mathbf{e}}$ es la cantidad adsorbida por la biomasa en $(\mathrm{mg} / \mathrm{g}), \mathbf{C}_{\mathbf{0}}$ es la concentración inicial de los metales, $\mathbf{C}_{\mathbf{e}}$ es la concentración de los iones $\mathrm{Pb}$ (II) y $\mathrm{Cd}$ (II) en el equilibrio, $\mathbf{V}$ es el volumen de la solución (L) y $\mathrm{W}$ es la cantidad de adsorbente (g), el experimento se realizó a $30^{\circ} \mathrm{C}$ (Yi y Shui 2011).

Los datos de adsorción para las concentraciones de adsorbato fueron descritos por los modelos de isotermas Langmuir y Freundlich. El modelo Langmuir se describe según la ecuación 2 y el modelo Freundlich por la ecuación 3.

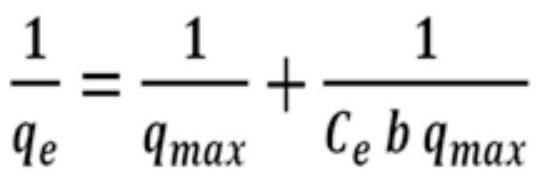

Ecuación 2.

$$
\log \left(q_{e}\right)=\log \left(K_{f}\right)+\frac{1}{n} \log \left(C_{e}\right)
$$

Ecuación 3.

Donde $\mathbf{q}_{\mathbf{e}}$ es la cantidad adsorbida por la biomasa en $(\mathrm{mg} / \mathrm{g}), \mathbf{C}_{\mathbf{e}}$ es la concentración de los iones en el equilibrio, $\mathbf{q}_{\max }$ representa la cantidad máxima adsorbida, $\mathbf{b}$ es la afinidad entre biosorbente $y$ biosorbato, $\mathbf{K}_{\mathbf{f}} \mathrm{y}^{\mathbf{1}}{ }_{\mathbf{n}}$ son las constantes de isoterma Freundlich (Yi y Shui 2011).

\section{Valoración de los sitios de unión metal- biomasa}

Se analiza preparando un stock mixto de $\mathrm{Pb}$ (II) y Cd (II) a 51,5 $\mathrm{mgL}^{-1}$ y $0,5 \mathrm{~g}$ de biosorbente, después de 24 horas, posteriormente la biomasa se secó a temperatura ambiente. Los espectros IR se realizaron utilizando un espectrofotómetro FT-IR Nicolet Is5 THERMO SCIENTIFIC en la región de 500-4000 $\mathrm{cm}^{-1}$ (Natarajan et al. 2010).

\section{RESULTADOS Y DISCUSIÓN}

\section{Caracterización de cepas fúngicas de Penicillium sp}

El hongo presentó colonias blancas, aterciopeladas, ya avanzado en tiempo de crecimiento presentó una coloración verdosa oscura, no generó pigmentos en los medios de crecimiento. Mostró formaciones concéntricas, conservando su anverso de color blanco. Sus hifas vegetativas, formadas por simple división, dieron origen a métulas. Cada métula dio origen a tres fiálides que terminaban en 
células conidiógenas o conidióforos, que a su vez, dieron origen a conidios. Se observa su peculiar forma de "cabeza de cepillo". Sus conidios son de forma oval y que presentaron desprendimiento a manera de cadena, lo que confirma que positivamente se trata de la especie Penicillium sp. (Valencia 2004).

Efecto de la concentración inicial de metales, pH y temperatura en la remoción de metales por Penicillium sp

En el efecto de la concentración inicial de metales se muestra en la figura 1 que grafica él porcentaje de adsorción de $\mathrm{Pb}$ (II) y Cd (II) en los distintos niveles de concentración.

Para el Pb (II) los más altos niveles de remoción se hallan a las concentraciones 51,5 y 103 mg $\mathrm{L}^{-1}$ con porcentajes de 62,9 y $60,4 \%$ respectivamente. En el caso del Cd (II) las más altas remociones se encontraron en niveles de concentración de 30 y 51,5 mg L-1 con porcentajes respectivos de 12,4 y $14,6 \%$.
A medida que aumentan las concentraciones de los metales en la solución la capacidad de adsorción se va manteniendo constante, debido a la saturación de los sitios disponibles en la superficie del biosorbente (Volesky y Holan 1995).

En general en el nivel de 51,5 $\mathrm{mg} \mathrm{L}^{-1}$ corresponde a la concentración adecuada en que se pudo encontrar los mayores porcentajes de remoción de los dos contaminantes tóxicos. Los diferentes porcentajes de adsorción para los contaminantes muestran que el biosorbente adsorbe en una mayor cantidad los iones $\mathrm{Pb}$ (II) que $\mathrm{Cd}$ (II).

Para el efecto $\mathrm{pH}$ la máxima eliminación de $\mathrm{Pb}$ (II) se observó entre pH 6 y pH 10 de acuerdo con la figura 2, la biosorción de $\mathrm{Pb}$ (II) por Penicillium sp. aumentó gradualmente de $\mathrm{pH}$ 2 a pH 5 y se estabilizó en 6. Para el Cd (II) la adsorción aumenta con el pH del sistema, el aumento se aprecia entre los valores de 3 a 5 ,

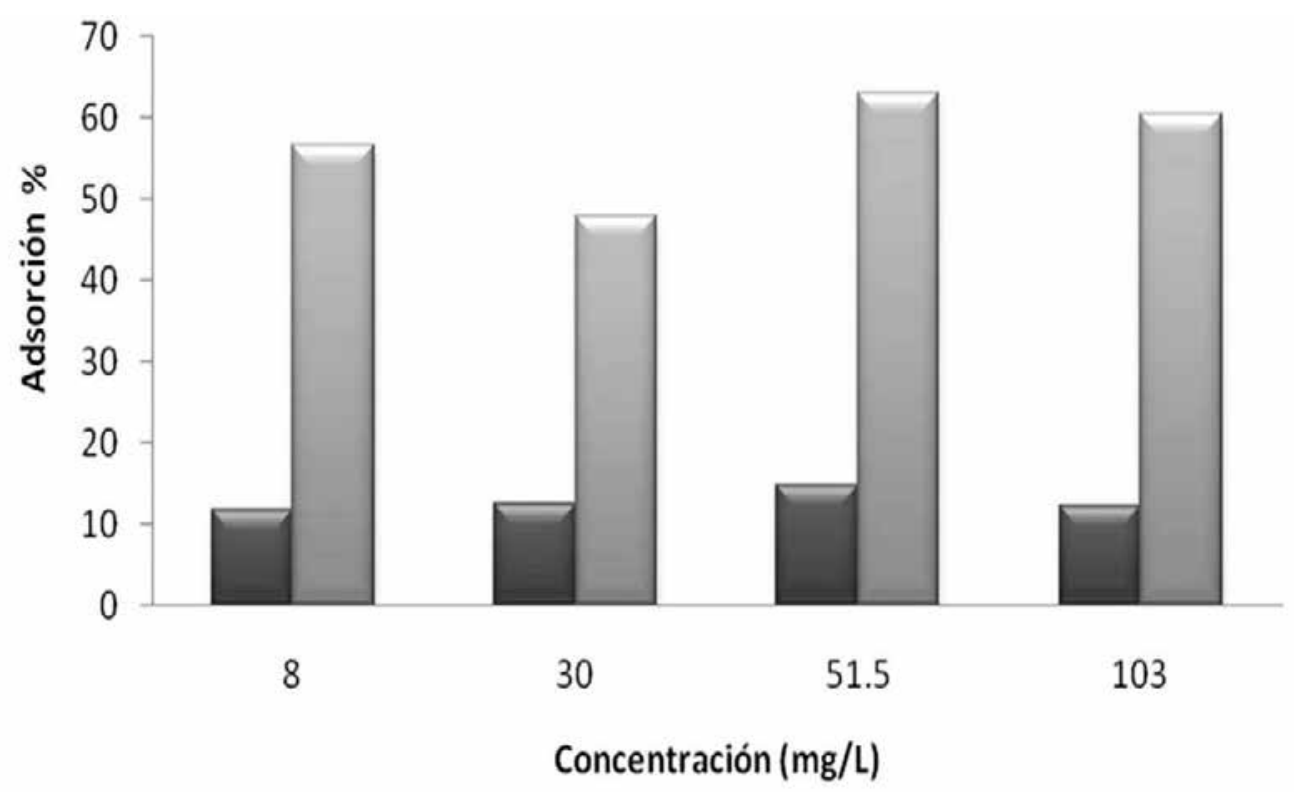

Figura 1. Efecto de la concentración inicial de metales tóxicos en la Adsorción por Penicillium sp. 
alcanzando su máxima eliminación a pH 6 y una posterior estabilización de 7 a 10.

Así, las adsorciones más altas se lograron en un $\mathrm{pH}$ ácido (4-5), pudiéndose apreciar que se reduce en pHs alcalinos. A pH bajo, en la superficie del hongo las cargas positivas impiden el contacto de los iones metálicos y la superficie cargada negativamente (Gupta y Rastogi 2008). Sin embargo, el aumento del pH entre 4 y 5 aumenta la eliminación de los iones metálicos disponibles en la solución, porque la neutralización de las cargas positivas en la superficie de hongo, mejora el contacto entre los iones metálicos y la superficie. Por encima de $\mathrm{pH} 7$ los iones metálicos precipitan en la solución y se inhibe el contacto entre la carga negativa de la superficie del hongo y los iones metálicos (Gupta y Rastogi 2008). Por lo tanto, la eliminación de iones metálicos se estabiliza por encima de $\mathrm{pH}$ 7. Similares resultados fueron publicados por Fan y colaboradores (2008) para la eliminación de Pb (II) mediante el uso de Penicillium simplicissimum. Yan y
Viraragavan (2003) también informaron que la eliminación de $\mathrm{Pb}$ (II) por Penicillium digitatum y Rhizopus nigricans, disminuía con disminuir el $\mathrm{pH}$ y aumenta con el aumento del mismo.

Los resultados del efecto temperatura se resumen en la figura 3, donde la relación de adsorción de metales por unidad de biomasa aumento con la temperatura para todos los metales logrando valores máximos a $60^{\circ} \mathrm{C}$.

Resultados similares se reportaron por Fan y colaboradores (2008), que mostraban la biosorción de plomo, cadmio y zinc con el hongo Penicillium simplicissimum realizando ensayos desde 20 a $40^{\circ} \mathrm{C}$. Llegaron a la conclusión que la adsorción de $\mathrm{Pb}$ (II) era endotérmica. La energía dependiente de la eliminación de metales por los microorganismos se ve influenciada en gran medida por la temperatura, ya que el proceso de adsorción es fisicoquímico. Mientras tanto, Mishra y Roy (1996) informaron que la adsorción de Zn (II) por Penicillium sp. disminuye con el aumento

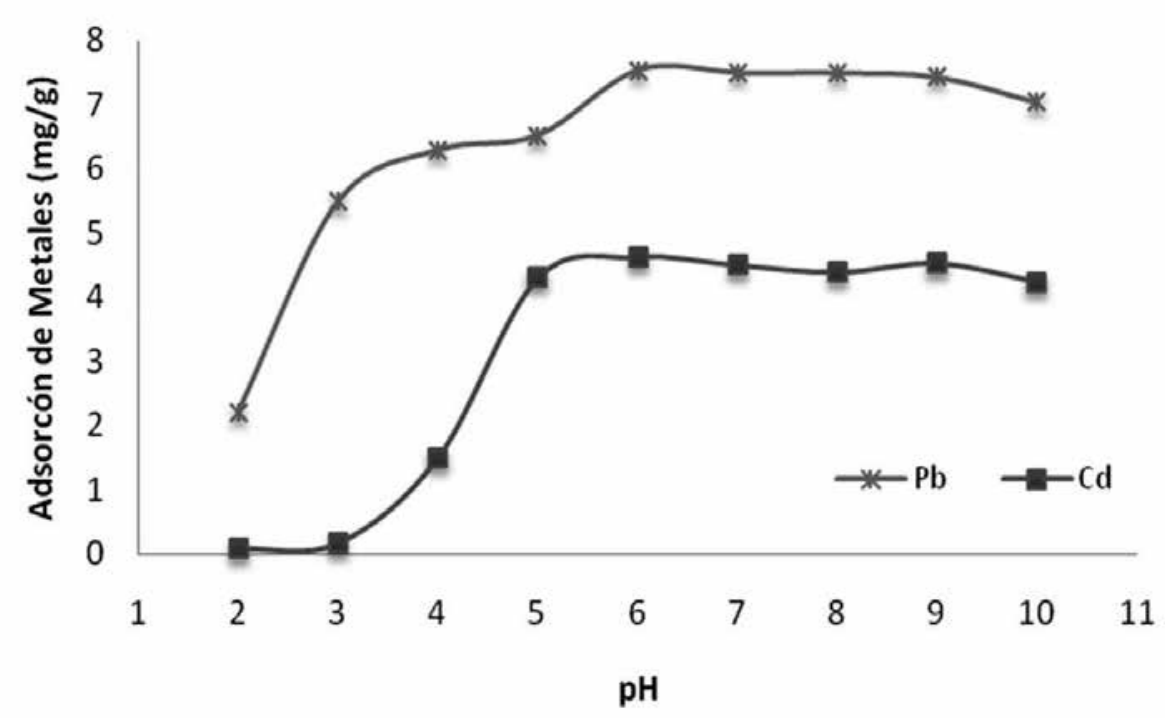

Figura 2. Efecto del pH en la Adsorción de Pb (II) y Cd (II) a 51,5 mg/L con biomasa de Penicillium sp. 


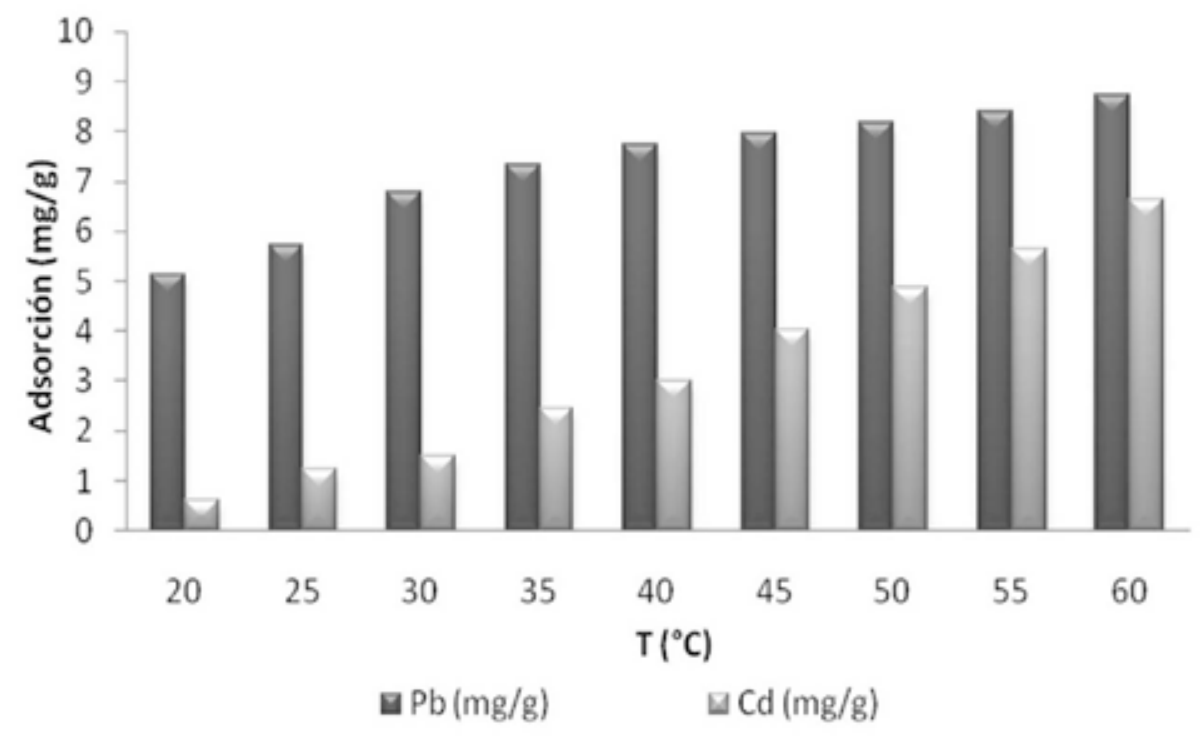

Figura 3. Efecto de la temperatura $\left({ }^{\circ} \mathrm{C}\right.$ ) en la Adsorción de $\mathrm{Pb}$ (II) y Cd (II) a $51.5 \mathrm{mg} / \mathrm{L}$ con biomasa de Penicillium sp.

de temperatura, debido a que la temperatura puede afectar la estabilidad de la pared celular de los hongos, lo que lleva a la hidrólisis de la pared celular. De acuerdo con el informe de Fan et al (2008), la adsorción de Pb (II) por Penicillium sp. era endotérmica. Realizando el respectivo control de calidad con el análisis por triplicado de muestras de material certificado de referencia NIST; SRM 1643 e para Pb y Cd para aguas naturales, se evaluó la exactitud como \% de error $(\% \mathrm{E})$ y la precisión como coeficiente de variación (CV), arrojando para $\mathrm{Pb}$ un $\mathrm{CV}$ de 0,613 y \% $\mathrm{E}$ de 0,582, para $\mathrm{Cd}$ se obtuvo un CV de 0,752 y un \%E de 0,238, encontrándose entre los criterios de calidad analítica (Miller y Miller 2002).

\section{Estudios de Isotermas de adsorción}

Los resultados de isotermas Langmuir $y$ Freundlich para hongos Penicillium sp. a $30^{\circ} \mathrm{C}$ se muestran en la tabla 1.

las constantes obtenidas de las isotermas Langmuir y Freundlich tuvieron coeficientes de correlación $\left(\mathrm{R}^{2}\right)$ altos. Las comparaciones entre los coeficientes de correlación para isotermas Langmuir y Freundlich con biomasa de hongos Penicillium sp. se ajustan mejor al modelo de Langmuir que al modelo de Freundlich, ya que los valores $\mathrm{R}^{2}$ para Langmuir son más altos que los $\mathrm{R}^{2}$ Freundlich, además los valores de las constantes de energía de adsorción b están muy cercanos a cero, indicando esto que el modelo de isoterma para este estudio está más fuertemente soportado por isotermas Langmuir que por isoterma Freundlich, lo que sugiere que todos los centros activos de adsorción en la biomasa fúngica son equivalentes y que la capacidad de los metales para unirse a la superficie es independiente de si hay o no posiciones próximas ocupadas. Además, la adsorción se restringe a una monocapa y no existen interacciones laterales entre los iones metálicos. Los valores b indican la afinidad de un biosorbente hacia un adsorbato, a medida que sean más pequeño el biosorbente tienes más afinidad hacia el metal (Natarajan et al 2010), por ende el biosorbente que tiene más 
afinidad hacia el $\mathrm{Pb}$ (II) con valor b de $0,0030 \mathrm{~L}$ $\mathrm{mg}^{-1}$, seguido del $\mathrm{Cd}$ (II) con valor b de 0,0031 $\mathrm{L} \mathrm{mg}^{-1}$. Los $\mathrm{q}_{\max }$ que representa la máxima capacidad de biosorción del biosorbente para cada metal son muy diferentes, sobresaliendo el $\mathrm{Pb}$ (II) con el valor más alto $\left(83,333 \mathrm{mg} \mathrm{g}^{-1}\right)$, seguido Cd (II) con un qmax de 8,696 $\mathrm{mg} \mathrm{g}^{-1}$, lo que sugiere que la capacidad máxima de adsorción para los tres metales recae sobre el $\mathrm{Pb}$ (II). Para analizar los resultados con el Freundlich, el más alto valor $\mathrm{K}_{\mathrm{f}}$ se halló para $\mathrm{Cd}$ (II). Un alto de $\mathrm{K}_{\mathrm{f}}$ Indica un volumen alto de adsorción y un alto valor $1 / n(n>1)$ indica una alta fuerza de adsorción. Así el más alto valor n fue hallado para Cd (II) con 0,961 seguido de $\mathrm{Pb}$ (II) con 0,939. Resultados similares fueron reportados por Fan y colaboradores (2008) en la remoción de Pb (II) por Penicillium Simplicissium.

\section{Valoración de los sitios de unión metal- biomasa}

La figura 4 muestra el espectro FT-IR de la biomasa del hongo Penicillium sp. antes (control) y después del tratamiento de remoción de los metales pesados. La estructura de la superficie de los hongos se infiere con los grupos funcionales encontrados en los espectros, mostrando los grupos activos del Penicillium sp. a la hora de la remoción. Se observan en la muestra control grupos en (3396), (2925), (1654), (1548) y (1150) $\mathrm{cm}^{-1}$.

Tabla 1. Modelos de isotermas Langmuir y Freundlich de la adsorción de Cd (II) y Pb (II).

\begin{tabular}{ccccccccc}
\hline & \multicolumn{9}{c}{ Isotermas } \\
\cline { 3 - 8 } Metal & \multicolumn{3}{c}{ Langmuir } & \multicolumn{3}{c}{ Freundlich } \\
& & & & & & & \\
& & $\mathrm{q}_{\max }$ & $\mathrm{b}$ & $\mathrm{R}^{2}$ & $\mathrm{~K}_{\mathrm{f}}$ & $\mathrm{n}$ & $\mathrm{R}^{2}$ \\
$\mathrm{Cd}(\mathrm{II})$ & 8.696 & 0.003 & 0.999 & 39.355 & 0.961 & 0.991 \\
$\mathrm{~Pb}(\mathrm{II})$ & 83.333 & 0.003 & 0.980 & 4.497 & 0.939 & 0.946 \\
\hline
\end{tabular}

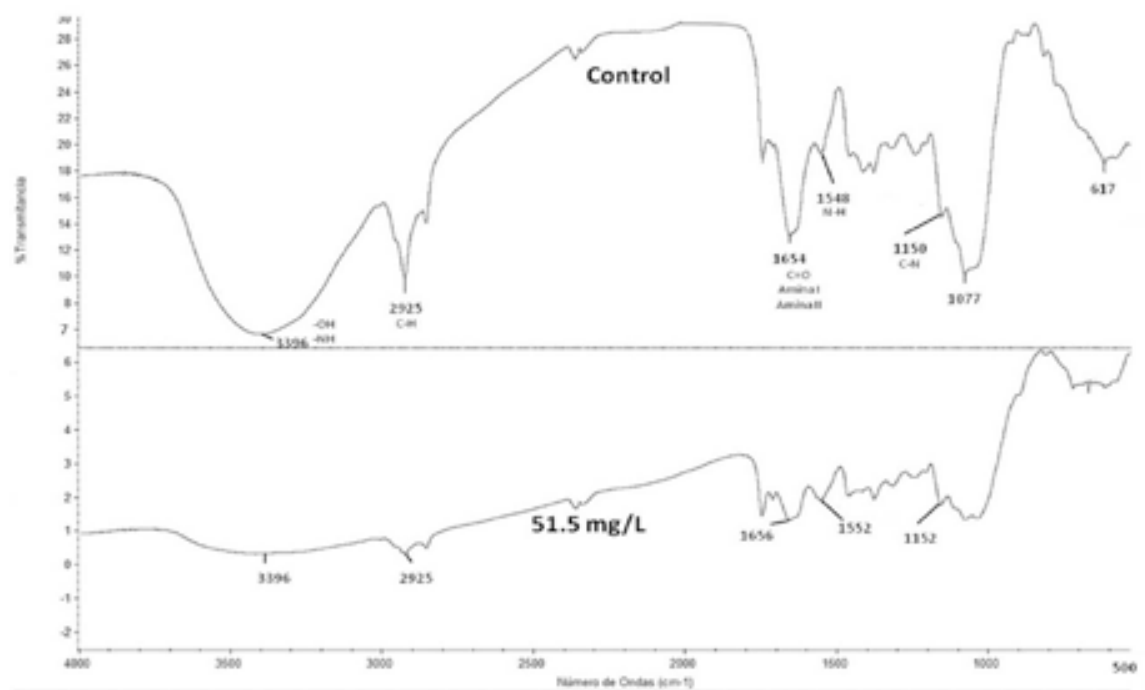

Figura 4. FT-IR de Biomasa de Penicillium sp. Antes de tratamiento (control) y después de tratamiento con $51,5 \mathrm{mg} / \mathrm{L}$ de $\mathrm{Pb}$ (II) y $\mathrm{Cd}(\mathrm{II})$. 
Un pico ancho observado entre 3200 a 3600 $\mathrm{cm}^{-1}$ fue atribuido a modos de tensión de grupos $-\mathrm{OH}$ y - NH. Apareció un pico fuerte en (1150) $\mathrm{cm}^{-1}$ indicando vibraciones de estiramiento C-N (Shringer et al. 1998). Enlaces C-H típico de estiramiento y vibraciones $\mathrm{N}-\mathrm{H}$ fueron asignados a picos en (2925) y (1548) $\mathrm{cm}^{-1}$. El pico fuerte en (1654) indica un grupo el estiramiento del grupo $\mathrm{C}=\mathrm{O}$ o amida I y amidas II (Dengy Ting 2005). La presencia de una estructura química común para polisacáridos se encontró entre 1200 a $800 \mathrm{~cm}^{-1}$ (Natarajan et al. 2010), y el pico a (1150) $\mathrm{cm}^{-1}$ es atribuido al estiramiento $\mathrm{C}-\mathrm{N}$ en la muestra control, es desplazado a (1152) $\mathrm{cm}^{-1}$ en la muestra tratada después de la absorción de metales. Los cambios presentes en la región de 1660 y 1500 $\mathrm{cm}^{-1}$ para $\mathrm{N}-\mathrm{H}$ también se debieron a conducir absorción (Dengy Ting 2005).

\section{CONCLUSIONES}

Un potencial biosorbente de metales pesados del tipo fúngico del genero Penicillium sp. fue aislado de suelos de mina contaminados por metales pesados. Los resultados experimentales mostraron una alta capacidad del Penicillium sp. para eliminar $\mathrm{Pb}$ (II) y $\mathrm{Cd}$ (II) en solución acuosa. La concentración inicial, el pH óptimo y condiciones de temperatura para la eliminación de $\mathrm{Pb}$ (II) y Cd (II) se determinó en el estudio. Los datos experimentales fueron analizados utilizando modelos de isotermas para evaluar el rendimiento del hongo biosorbente. El análisis IR muestra bandas y picos muy característicos a los enlaces de grupos funcionales $-\mathrm{OH},-\mathrm{NH}, \mathrm{C}-\mathrm{N}, \mathrm{C}-\mathrm{H}, \mathrm{N}-\mathrm{H}$, $\mathrm{C}=\mathrm{O}$, amida I y amidas II y polisacáridos, la capacidad de adsorción a los metales puede ser atribuida a que la gran mayoría tienen cargas parcialmente positivas que pueden generar la atracción entre los metales y la biomasa fúngica. Por último, los resultados indican que los hongos aislados de los suelos de mina, podrían ser utilizados como un biosorbente rentable y fácilmente cultivable para la eliminación de los iones metálicos de ambientes contaminados de metales pesados.

\section{AGRADECIMIENTOS}

A la universidad de Córdoba y a Colciencias por la financiación de este proyecto contrato 223-2010.

\section{REFERENCIAS}

Agarwal, G., Bhuptawat, H. and Chaudhari, S. 2006. Biosorption of aqueous chromium (VI) by tamarindus indica seeds. Bioresource Technology. 97: 949-956.

Deng, L., Su, Y., Su, H., Wang, X. and Zhu, X. 2006. Biosorption of copper(II) and lead(II) from aqueous solutions by nonliving green algae Cladophora $f$ ascicularis: equilibrium, kinetics and environmental effects. Adsorption. 2: 267-277.

Deng, S. and Ting, P. 2005. Characterization of PEl-modified biomass and Biosorption $\mathrm{Cu}(\mathrm{II}), \mathrm{Pb}(\mathrm{II})$ and $\mathrm{Ni}(\mathrm{II})$. Water Research 39: 2167-2177.

Fan, T., Liu, Y., Feng, B., Zeng, G., Yang, C. and Zhou, M. 2008. Biosorption of cadmium (II), zinc (II) and lead (II) by Penicillium simplicissimum: Isotherms, kinetics and thermodynamics. Journal Of Hazardous Materials. 160: 655-661. 
Gupta, V. and Rastogi, A. 2008. Biosorption of lead from aqueous solutions by green algae Spirogyra species: kinetics and equilibrium studies. Journal Of Hazardous Materials. 152: 407-414.

Johannes, F., Grosse, C., Esche, V., Brandenburg, P., Reich, A. and Groneberg, D. 2006. The toxicity of cadmium and resulting hazards for human health.Journal of Occupational Medicine and Toxicology. 1: 1-6.

Lin, L., Li, J. and Juang, R. 2008. Removal of $\mathrm{Cu}$ (II) and $\mathrm{Ni}$ (II) from aqueous solutions using batch and fixed-bed ion exchange processes. Desalination. 225: 249-259.

Miller, J., y Miller, J. 2002. Exactitud. In Estadística y Quimiometría para Química Analítica. Prentice Hall, Madrid. p277-278.

Mishra, S. and Roy, C. 1996. Kinetics of $\mathrm{Zn}+2$ adsorption by Pencillium sp. Hydrometallurgy. 40: 11-23.

Natarajan, V., Grim, H., Muthuswamy, S., Tae, C., Kui, L. and Byung, O. 2010. Isolation, identification, $\mathrm{Pb}$ (II) biosorption isotherms and kinetics of a lead adsorbing Penicillium sp. MRF-1 from South Korean mine soil. Journal Of Environmental Sciences-China. 7: 1049-1056.
Pino, G., Mesquita, L. and Torem, M. 2006. Biosorption of heavy metals by powder of green coconut shell. Separation Science And Technology. 41: 3141-3153.

Shringer, R., Hermann, C., Morrill, T., Curtin, D. and Fuson, R. 1998. The Systematic Identification of Organic Compounds. Wiley, New York. 324 p.

Valencia, H. 2004. Cultivo, microcultivo e identificación de hongos. Manual de prácticas de microbiología básica. Facultad de Ciencias de la Universidad Nacional de Colombia, Bogotá. p99-116

Volesky, B. and Holan, Z. 1995. Biosorption of heavy metals.Biotechnology Progress. 11: 235-250

Yan, G. and Viraraghavan, T. 2003. Heavy metal removal from aqueous solution by fungus Mucorrouxii.Water Research. 37: 4486-4496.

Yi,L. and Shui, C. 2011. The biosorption of heavy metals from aqueous solution by Spirogyraand Cladophora filamentous macroalgae. Bioresource Technology. 102: 5297-5304.

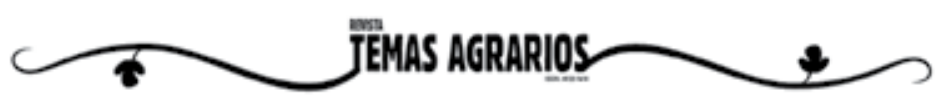

